NOTICE: this is the author's version of a work that was accepted for publication in Geochimica et Cosmochimica Acta. Changes resulting from the publishing process, such as peer review, editing, corrections, structural formatting, and other quality control mechanisms may not be reflected in this document. Changes may have been made to this work since it was submitted for publication. A definitive version was subsequently published in Geochimica et Cosmochimica Acta, Vol. 91 (2012) http://dx.doi.org/10.1016/j.gca.2012.05.034 


\title{
Lithium defects and diffusivity in Forsterite
}

\author{
Feiwu Zhang and Kate Wright \\ Nanochemistry Research Institute, Curtin University, PO Box U1987, \\ Perth, WA 6845, Australia
}

\begin{abstract}
Lithium is an important geochemical tracer used to infer the thermal and chemical evolution of minerals in the Earth's upper mantle. Knowledge of point defect chemistry and diffusion is critical for the interpretation of Li distribution in minerals. Using quantum mechanical methods we show that in forsterite Li will be incorporated as bound interstitial-substitutional pairs. Furthermore, there will be temperature dependent fractionation of its two isotopes between the different sites. The fractionation decreases dramatically from $87.1 \%$ at $300 \mathrm{~K}$ to $1.0 \%$ at $3000 \mathrm{~K}$. Diffusion is predicted to occur via two inter-related mechanisms: $\mathrm{Mg}$ - $\mathrm{Li}$ exchange, and a second, vacancy assisted interstitial mechanism. This behaviour is complex, facilitates migration of the heavier isotope and offers insights into observations of $\mathrm{Li}$ mobility and zoning in olivine, the most volumetrically important upper mantle mineral.
\end{abstract}

\section{INTRODUCTION}

The distribution of impurities in minerals can provide important clues to thermal and chemical processes occurring in the Earth's mantle over geological time. Lithium (Li) is increasingly used as a geochemical tracer as its two isotopes $\left({ }^{6} \mathrm{Li}\right.$ and $\left.{ }^{7} \mathrm{Li}\right)$ have a large mass difference and thus fractionation can lead to compositional variations at 
low temperatures. Within the Earth's mantle, the largely incompatible Li is expected to be hosted by olivine $\left[(\mathrm{MgFe})_{2} \mathrm{SiO}_{4}\right]$ (OTtolini et al., 2004; SEITZ and WoodLAnd, 2000) and can provide insights into changing conditions during crustal recycling processes (ELLIOTT et al., 2004). The mechanism by which Li and other monovalent cations are incorporated into olivine and their diffusivities however, are poorly understood. Yet this information is critical if we are to fully interpret chemical signatures and model processes occurring over long timescales. Recent research has shown that the distribution of $\mathrm{Li}$ isotopes in co-existing phases such as olivine and pyroxene, can vary greatly (e.g. JEFFCOATE et al., 2007; MAGNA et al., 2006) and, in addition, there can be major variations within a single grain. Further complications arise in response to "matrix effects" described by Bell et al (2009), resulting from small chemical variations that change local environments in the crystal lattice. A chemical atomic-level understanding of defect and diffusion processes in minerals such as olivine is therefore needed.

Recent experiments (DOHMEN et al., 2010) provided the first real clues as to the controls on Li diffusion rates in natural olivines, although the mechanisms responsible for diffusion are hard to interpret unambiguously. We have carried out calculations, primarily using first principles methods, to model the behaviour of $\mathrm{Li}$ in forsterite, the $\mathrm{Mg}$ end member olivine. Our results complement those from a recent experiment and provide an insight into processes occurring at the atomic level, not accessible by other means. The present work focuses on $\mathrm{Li}$ point defects and diffusion. It provides quantitative estimates of the stability of $\mathrm{Li}$ in forsterite, and the corresponding substitution and migration mechanisms. Additionally, we investigate the possibility of $\mathrm{Li}$ isotopic site fractionation. The results enhance our knowledge of the behavior of 
chemical exchange, material transport and electrical conductivity in the Earth's upper mantle, and to interpret observed isotopic variations in real rocks.

\section{METHODOLOGY}

Based on density functional theory (DFT), our ab initio simulations were performed with the CASTEP code (CLARK et al., 2005; SEgall et al., 2002). The Perdew Burke Ernzerhof (PBE) functional was used with the generalized gradient approximation (GGA) for all calculations (PERDEW et al., 1996). Ultrasoft pseudopotentials that we used have the valence-electron configurations of $1 \mathrm{~s}^{2} 2 \mathrm{~s}^{1}$ (core radius 1.86 a.u.) for $\mathrm{Li}$, $2 \mathrm{p}^{6} 3 \mathrm{~s}^{2}$ (core radius 2.06 a.u.) for $\mathrm{Mg}, 2 \mathrm{~s}^{2} 2 \mathrm{p}^{4}$ (core radius 1.0 a.u.) for $\mathrm{O}, 3 \mathrm{~s}^{2} 3 \mathrm{p}^{2}$ (core

radius 1.8 a.u.) for Si, respectively. 3 x 1 x 2 supercell $(14.3 \AA \times 10.3 \AA \times 12.1 \AA$ and $90.0^{\circ}, 90.0^{\circ}, 90.0^{\circ}$ ) with 168 atoms was used for the defect calculations and we have used the plane-wave cut-off of $700 \mathrm{eV}$ for representing the wave functions and only $\Gamma$-point for the Brillouin zone sampling. Geometry optimizations on the defective supercells were performed without imposed symmetry using the BFGS procedure (Pfrommer et al., 1997) at $0 \mathrm{~K}$. The energy tolerance was $5 \times 10^{-6} \mathrm{eV} / \mathrm{atom}$; the maximum tolerance for force and atom displacements along any Cartesian component was $0.01 \mathrm{eV} / \AA$ and $5 \times 10^{-4} \AA$, respectively.

\subsection{Isotope fractionation}

Within the forsterite structure, the fraction of the two Li isotopes at substitutional and interstitial sites is represented by the following:

$\alpha_{\text {interstitial-vacancy }}=\frac{\left[{ }^{7} \mathrm{Li} /{ }^{6} \mathrm{Li}\right]_{\text {interstitial }}}{\left[{ }^{7} \mathrm{Li} /{ }^{6} \mathrm{Li}\right]_{\text {vacancy }}}=\frac{\beta_{\text {interstitial }}}{\beta_{\text {vacancy }}}$ 
where $\alpha_{\text {interstitial-vacancy }}$ is the isotope fractionation factor (also known as separation or enrichment factor) for interstitial and vacancy substitution mechanisms, and $\beta$ is the mass-dependent isotope partition function ratio, which can be estimated by the simplified formula (BIGELEISEN and MAYER, 1947):

$\beta=\frac{{ }^{7} L i}{{ }^{6} L i}=\prod_{i=1}^{N} \frac{U_{i}^{*}}{U_{i}} \exp \left(\frac{U_{i}-U_{i}^{*}}{2}\right) \frac{1-\exp \left(-U_{i}\right)}{1-\exp \left(-U_{i}^{*}\right)}$

Here, $\mathrm{N}$ represents the number of vibrational frequencies in the system; $U_{i}=h v / K_{B} T$ for the $i^{\text {th }}$ vibrational mode, $h$ is Planck's constant, $v$ represents the vibrational frequency, $K_{B}$ is Boltzmann's constant, $T$ is the temperature in Kelvin, and $U i^{*}$ is the analogous term for the system where one atom is substituted with a heavier isotope. Therefore, the isotope fractionation factor $\alpha$ only relates to the harmonic vibrational frequencies associated with the isotopic substitution. The methods described here have been successfully employed to model isotopic fractionation of $\mathrm{Fe}$ and $\mathrm{Ge}$ in a range of materials (ANBAR et al., 2005; BLANCHARD et al., 2009; LI et al., 2009).

Vibrational frequencies for the system, and the effect of isotope substitution on those frequencies, were calculated using ab-initio lattice dynamics methods. The lattice dynamical properties are calculated within the framework of self-consistent densityfunctional perturbation theory (DFPT). The calculations of the lattice dynamics properties are based on the same ultrasoft pseudopotentials and energy cutoff used for the defect stability investigation. However, for both accuracy and efficiency purposes, one formula unit cell 28 atoms was used for the phonon calculations and 8 × 4 x 8 Monkhorst-Pack k-point mesh with $128 k$-points grid was used for the frequencies calculations. During the simulation, the vibrational frequencies are calculated numerically by calculating forces acting due to small displacements of the atoms. The lattice dynamics 
simulations are performed on the highly converged and optimized geometry of forsterite with either interstitial or substitutional $\mathrm{Li}$ isotopic defects.

\subsection{Defect calculations}

Point defects are introduced into an optimised forsterite supercell $(3 \times 1 \times 2)$ by removing $\mathrm{Mg}$ to create vacancies, by replacing $\mathrm{Mg}$ with $\mathrm{Li}$, or putting $\mathrm{Li}$ into an interstitial site. The atomic positions are again relaxed to find an energy minimum representing the Li point defects in the lattice. We are interested in how the energetic cost of introducing the Li impurity varies with its location in the structure, i.e. the substitution mechanism. To find the most energetically favourable Li substitution mechanism, we performed a search over different sites for Li point defects. The periodic nature of the supercells means that for charged defects, there will be a defectdefect interaction contribution to the total energy of the system. In some cases this can be significant and thus we need to evaluate and correct for, this energy term. In this work, unwanted defect-defect interactions have been corrected using the methodology described in (BRODHOLT and REFSON, 2000). To measure the point defect stability, we calculate the supercell energies with various defect configurations and compare the differences between them.

The mobility of Li point defects along the diffusion path is determined by moving a Li ion in small steps from an initial low energy start point, to an equivalent low energy end point, where the start and end points can be substitutional or interstitial sites. The geometry is relaxed at each step under the constraint that the migrating ion does not move in the direction parallel to the diffusion path (i.e. it is fixed in this direction) but can relax perpendicular to the line. Plotting a graph of energy against interstitial displacement shows the energy barrier to diffusion by either a vacancy or 
interstitial mechanisms. The difference between the energy minimum and maximum on this pathway is the energy barrier to migration, ignoring the energy cost of creating the point defect.

\section{RESULTS AND DISCUSSION}

\subsection{Defect species}

Li can be incorporated into the pure forsterite lattice as either an interstitial or as a substitutional defect at an $\mathrm{Mg}$ site, both of which require charge compensation. In Kröger-Vink defect notation these are denoted $\left(L i_{i}^{*},\right)$ and $\left(L i_{M g}^{\prime}\right)$, for the interstitial and substitutional configurations respectively. Charge neutrality of Li incorporated in the pure forsterite lattice involves combinations of interstitials and substitutions that are described as:

(a) $L i_{i}+L i_{M g}^{\prime}$, where the interstitial is isolated from the substitution, or,

(b) $2 L i_{i}+V_{M g}^{\prime \prime}$, where the two interstitial ions are displaced around the vacancy $\left(V_{M_{g}}^{J}\right)$ as a "split interstitial". The calculated energies of all configuration componets of (a) and (b), and the lattice energies of associated phases are provided in Table 1.

The first incorporation mechanism (a) involves Li substitution at an $\mathrm{Mg}$ site, plus a $\mathrm{Li}$ interstitial. Forsterite has two crystallographically distinct Mg sites, M1 and M2. Our calculations indicate that Li substitution is energetically favoured at the M1 site by 47 $\mathrm{kJmol}^{-1}$. We note that the formation of a $\mathrm{Mg}$ vacancy in pure forsterite is $99 \mathrm{kJmol}^{-1}$ lower at M1 compared to M2, which is agree with the previous studies (BRODHOLT, 1997; BRODHOLt and RefSON, 2000). The incorporation of Li interstitials in an otherwise perfect lattice was thoroughly explored to locate the minimum energy 
position within the structure. This is indicated on Figure 1a as point I, adjacent to an M2 site. A second position, only $3.8 \mathrm{kJmol}^{-1}$ higher than the first, is found at position $\mathrm{S}$ and $\mathrm{Li}$ can move easily between the two sites. At point $\mathrm{S}$, the presence of the interstitial displaces the $\mathrm{Mg} 1$ from its site and this may be important for migration as detailed below. To investigate the partitioning of Li between the low energy interstitial site and substitutional sites, we consider the following equation:

$$
\left.L i_{i}^{\bullet} 0 V_{M g}^{\prime \prime}\right\rangle L i_{M g}^{\prime}
$$

To calculate the energy we expand this expression and balance the reaction as:

$$
\left.\mathrm{Mg}_{2} \mathrm{Li}_{x} \mathrm{SiO}_{4} \mathrm{O} \mathrm{Mg}_{22 x} \mathrm{SiO}_{4}\right\rangle \mathrm{Mg}_{2} \mathrm{SiO}_{4} \mathrm{O} \mathrm{Mg}_{22 x} \mathrm{Li}_{x} \mathrm{SiO}_{4}
$$

and compute the associated energy using the values in Table 1. Not surprisingly, the right hand side of Equations $4 \mathrm{a}$ and $4 \mathrm{~b}$ represents the more stable configuration by $148 \mathrm{kJmol}^{-1}$, and thus Li will partition to vacant metal sites in forsterite..

The second Li incorporation mechanism, with the split interstitial, has Li ions at $\mathrm{S}$ and S' (see Figure 1), balanced either side of a Mg vacancy. The Li split interstitial complex is charge neutral and can occur about either $\mathrm{Mg} 1$ or $\mathrm{Mg} 2$. As before, we find that the configuration involving the $\mathrm{Mg} 1$ vacancy is the more energetically favorable by $55 \mathrm{kJmol}^{-1}$. We can determine if mechanism (a) or (b) is the more thermodynamically stable complex by considering the relative energies of the two configurations:

$$
\left(2 L i_{i}+V_{M g}\right)_{S p l i t}^{x} \Leftrightarrow L i_{i}^{*}+L i_{M g}^{f}
$$

As before, the balanced reaction is written as:

$$
\left.\mathrm{Mg}_{22 x} \mathrm{Li}_{2 x} \mathrm{SiO}_{4} \mathrm{O} \mathrm{Mg}_{2} \mathrm{SiO}_{4}\right\rangle \mathrm{Mg}_{2} \mathrm{Li}_{x} \mathrm{SiO}_{4} \mathrm{OMg}_{22 x} \mathrm{Li}_{x} \mathrm{SiO}_{4}
$$

The computed energy is $-536 \mathrm{kJmol}^{-1}$, showing that the right hand side of Equation 5 is strongly favoured. Thus the split interstitial (substitution b) is far less stable than 
the separated interstitial and substitution (substitution a), and we have two distinct Li defect species present in otherwise pure forsterite as postulated by Dohmen (DOHMEN et al., 2010), and Grant and Wood (GRANT and WoOD, 2010).

In this study we consider the incorporation mechanism of $\mathrm{Li}$ into an otherwise pure forsterite, however in natural olivine, with its variable trace and minor element chemistry, the possibilities for charge compensation are much more varied. Coupled substitution of Li with trivalent cations (e.g. $\mathrm{Fe}^{3+}, \mathrm{Al}^{3+}$ or REE) in particular could be important. Our previous work (ZHANG and Wright, 2010; ZHANG and Wright, 2012), along with that of Purton et al.(1997), has shown that trivalent cations preferentially substitute onto the $\mathrm{Mg} 2$ site, charge balanced by $\mathrm{M}^{+}$ions such as $\mathrm{H}^{+}$and $\mathrm{Li}^{+}$on the $\mathrm{Mg} 1$ site. Where the compensating ion is $\mathrm{H}^{+}$, then a complex involving $\mathrm{M}^{3+}$ at a Si site with an accompanying hydroxyl group is also possible, particularly for $\mathrm{M}^{3+}$ cations with small ionic radii (ZHANG and WRIGHT, 2010). However, The $\mathrm{Li}^{+} / \mathrm{Al}^{3+}$ couple is extremely stable and appears to inhibit uptake of other impurities such as $\mathrm{H}^{+}$ that favour alternative defect schemes (ZHANG and WRIGHT, 2012). It is likely therefore that the presence of trivalent cations in olivine will facilitate the uptake of Li.

\subsection{Isotopic Fractionation}

To determine the possible extent of Li fractionation in forsterite, we calculated the vibrational frequencies associated with each of the two isotopes at both defect sites: $\mathrm{Li}_{i}$ and $\mathrm{Li}_{M g 1}$. At $400 \mathrm{~K}$, the mass-dependent isotope partition function ratios $(\cap)$, are calculated to be 1.0726 and 1.0201 for the interstitial and substitutional sites, respectively. Table 2 gives the calculated fractionation factors as a function of temperature, using the methods described in section 2. The largest fractionation occurs at low temperatures and decreases dramatically from $1.0871(87.1 \%)$ at $300 \mathrm{~K}$ 
to $1.001(1.0 \%)$ at $3000 \mathrm{~K}$. This demonstrates, as expected, that temperature is a key factor for $\mathrm{Li}$ isotope fractionation. These results (Table 2) suggest that heavy Li isotopes will be preferentially incorporated into forsterite via the interstitial mechanism, while the light $\mathrm{Li}$ isotopes will be enriched at $\mathrm{Mg}$ sites.

\subsection{Li Migration}

The interpretation of isotopic and chemical heterogeneities in minerals requires an understanding of diffusion behaviour in order to determine how and why such distributions may have changed over time. Commonly, diffusion is thought to take place by either a vacancy mechanism or via the migration of interstitial ions from one interstitial point to the next. In reality, the process may be more complex, involving clusters and correlated movements (TILLEY, 2008), particularly in the case of migrating heterovalent impurities.

Given that $\mathrm{Li}$ is present as both interstitial and substitutional defects, we have considered migration via $\mathrm{Mg}$ vacancies, via interstitial sites, and lastly via mixed interstitial/vacancy routes. For each, we calculated the activation barrier for migration which provides insights into the mechanisms responsible for diffusion. Using the current methods, however, we are not able to determine diffusion rates.

There are a number of possible jumps that $\mathrm{Li}$ can take as it diffuses through the forsterite lattice as illustrated in Figure 1a. Parallel to [001], we have two possible vacancy hopping routes: A involves vacancy hopping from $\mathrm{Mg} 2$ to $\mathrm{Mg} 2$ sites, and $\mathrm{D}$, from $\mathrm{Mg} 1$ to $\mathrm{Mg} 1$. Hops $\mathrm{B}$ and $\mathrm{B}$ ' between $\mathrm{Mg} 1$ and $\mathrm{Mg}$ 2, are mostly in the [100] plane, while the reverse ( $\mathrm{C}$ and $\mathrm{C}$ ') combines elements of all three principal crystallographic directions. The interstitial hops labeled E and F, relate to the [001] and $[010]$ directions respectively.

Table 3 gives the computed migration activation energy for each Li hop while the 
energy profiles are detailed in Figure 2. We note that these values represent the ion migration activation energies associated with the extrinsic diffusion regime, and thus do not include the energies associated with defect formation.

For Li migration by a pure vacancy mechanism, hop D along the [001] direction has the lowest activation energy, and hop C, from M2 to M1 has the highest, which reflects the high energy associated with a M2 vacancy. Our calculations show similar trends to those calculated by Walker et al. (2009) for Mg, found a low activation barrier in the [001] direction compared to [100] and [010] (see Table 3). In a pure system, when $\mathrm{Mg}$ ions are diffusing via a vacancy mechanism, there is a flow of ions in one direction and vacancies in the opposite direction, so that hops are continuous. With heterovalent dopant ions the situation is somewhat different, as once Li has taken up residence in the $\mathrm{Mg}$ site it can only move forward by exchanging with the next $\mathrm{Mg}$ on the migration pathway. This implies that Li migration rates via a vacancy mechanism will be dependent on (a) the Mg1 vacancy population and (b) by its capacity to exchange with Mg. Li-Mg exchange activation energy will not simply be a combination of the two jump energies but could be higher or lower, depending on the exact lattice interactions. The concurrent simulation of Mg-Li exchange is difficult as the actual exchange mechanism could be complex and involve a number of different stages. Techniques such as molecular dynamics can overcome such problem, although in this case the potential energy barrier is too high to be overcome within a realistic timeframe for such calculations. However, we can postulate that diffusion activation energies and rates for this mechanism will be controlled by the ability of the ions to exchange and that this will be reflected in any experimental measurements.

Moving to the interstitial mechanism, we see that Hop E which moves from the low 
energy interstitial site (I) to site (S) with almost no barrier $\left(3.8 \mathrm{~kJ} \mathrm{~mol}^{-1}\right)$. This value is within thermal energy and the limits of accuracy of the calculation and thus the path is essentially flat. Structurally, the presence of $\mathrm{Li}$ at the $(\mathrm{S})$ site displaces the $\mathrm{Mg} 1$ moving it to form a "split interstitial" type complex, with $\mathrm{Mg}$ and Li balanced either side of the vacancy. At this point, the simulations suggest two possible scenarios. In the first, the $\mathrm{Li}$ is in a stable position and is effectively pinned. It can only move further through the lattice by overcoming a very high activation barrier via hop $\mathrm{F}$ $\left(518.3 \mathrm{~kJ} \mathrm{~mol}^{-1}\right)$. However, if there is a $\mathrm{Mg}$ vacancy close by on either $\mathrm{Mg} 1$ or $\mathrm{Mg} 2$ sites, the displaced $\mathrm{Mg}$ will move into it and the Li will occupy the now vacant $\mathrm{Mg} 1$ site. This is shown schematically in Figure 1b-d. The barrier to move to the second interstitial site (S') via hop $F_{v}$ is $56 \mathrm{kJmol}^{-1}$ and thus we have a "vacancy assisted interstitial mechanism" that is also likely to be important in facilitating Mg migration along [001].

Experimental evidence relating to $\mathrm{Li}$ diffusion in forsterite or olivine is limited (Dohmen et al., 2010; PARKInSON et al., 2007; Spandler and O'NeILl, 2010). Activation energies obtained by Dohmen et al (2010) from modelling of their data are given as 240 and $220 \mathrm{kJmol}^{-1}$ for diffusion of the $\mathrm{Li}_{\mathrm{Mg}}$ and $\mathrm{Li}_{\mathrm{i}}$ species in the intrinsic (high temperature) regime. Comparison of calculated and experimental activation energies for such complex mechanisms is not straightforward. Within our calculations, we compute activation energies for individual hops, and these provide information on the most likely stages of the overall diffusion mechanism. In the experiment, the entire process is measured, and differentiating the different steps in such a co-operative process is problematic. In this case we believe that our simulations provide complementary information and an understanding of the 
atomistic scale processes that occur during diffusion.

Measured diffusion profiles of Li in olivine from diffusion experiments (DOHMEN et al., 2010) and from analyses of mantle olivine samples (PARKINSON et al., 2007), indicate that diffusion rates for ${ }^{6} \mathrm{Li}$ are slightly faster than ${ }^{7} \mathrm{Li}$. Our proposed model suggests that the lighter isotope will partition to the vacancy site and hence both ${ }^{7} \mathrm{Li}$ and ${ }^{6} \mathrm{Li}$ would be involved in the "mixed" migration mechanism. Additionally, Dohmen et al (2010) interpret their diffusion data in terms of a fast interstitial and a slower vacancy mechanism, working together in tandem. They conclude that the interstitial mechanism is unlikely to dominate behaviour but that overall the rate of $\mathrm{Li}$ diffusion will be an order of magnitude faster than $\mathrm{Fe}$ or $\mathrm{Mg}$ in olivine. This is in contrast to the study of Spandler and O'Neill (2010) whose experiments suggest that most minor elements in olivine diffuse at similar rates to the major cations, Fe and $\mathrm{Mg}$, regardless of ionic charge or radius. An analysis by Parkinson et al. (2007), suggests that Li diffusion is $4-8$ times slower than Fe-Mg diffusion in olivine but 20 - 30 times faster in co-existing clinopyroxene. We do not compute rates here, only activation energies for migration, and these suggest that Li diffusion via a purely interstitial mechanism is highly unlikely. Fast Li diffusion rates in minerals such as clinopyroxenes can occur because they contain channel structures that facilitate interstitial movement. The olivine structure does not. We believe that the presence of $\mathrm{Li}$ facilitates the formation of $\mathrm{Mg} 1$ vacancies and their migration; and that this in turn enhances Mg-Li exchange so that the overall barrier to diffusion is slightly reduced relative to $\mathrm{Mg}$ diffusion in pure forsterite. Indeed this is likely to be the case for most impurity species in olivine that substitute for $\mathrm{Mg}$ as suggested by Spandler and O’Neill (2010). 
Our results indicate that where temperatures are higher, e.g. in the deeper upper mantle, both Li isotopes will be able to migrate through the olivine structure, by way of the vacancy assisted interstitial diffusion mechanism. Rates will be influenced by Li concentration, co-existing mineral assemblages and the rate of $\mathrm{Mg}$ - $\mathrm{Li}$ exchange in olivine. However, at lower temperatures, ${ }^{7} \mathrm{Li}$ will fractionate to interstitial sites in the structure, and become trapped, while the migration of the more mobile ${ }^{6} \mathrm{Li}$ will be rate-limited by the $\mathrm{Mg}$ vacancy population.

Of course, as mentioned earlier, the presence of other impurities such as trivalent cations in natural systems could influence behaviour and facilitate the uptake of $\mathrm{Li}$ to provide charge balance. This would lead to changes in local structure around impurities (e.g. the matrix effects described by BELL et al., 2009) that could in turn lower the barriers to diffusion. However, given that concentrations of such impurities are generally low, their effects would be limited and on a macroscopic scale would not alter diffusion rates. Studies to investigate these relationships further are currently underway.

\section{CONCLUSIONS}

Lithium isotopes migrate and fractionate through forsterite via a complex vacancy assisted interstitial mechanism that has been simulated using computational methods. We have shown that the most stable route for incorporation of $\mathrm{Li}$ in the forsterite lattice is via formation of $\mathrm{Li}$ interstitial/substitutional pairs. The differential partitioning of $\mathrm{Li}$ isotopes between these two sites is the prime mechanism for $\mathrm{Li}$ isotopic fractionation in the major near-surface mineralogical Li reservoirs, which has 
significant implications for crust-mantle recycling at subduction zones. Our results suggest that pinning of interstitial ${ }^{7} \mathrm{Li}$ at low temperatures leads to fractionation and the low $\delta^{7} \mathrm{Li}$ observed in mantle-derived rocks. The Li diffusion pathway involves movement via both interstitial and $\mathrm{Mg}$ vacancy sites but is largely controlled by Li$\mathrm{Mg}$ exchange and by the concentration of $\mathrm{Mg}$ vacancies.

Acknowledgements. We thank the two anonymous reviewers, Dr. Ralf Dohmen and the associate editor, Dr. Michael Toplis, for their constructive comments. This work was carried out with the support of the Australian Research Council (DP0878453), with computational resources from iVEC and the NCI National Facility in Canberra, Australia, which are supported by the Australian Commonwealth Government. F.Z gratefully acknowledges Julian Gale for the fruitful discussion and help.

\section{REFERENCES}




Tables:

Table 1. Lattice energies for the perfect unit cells and the forsterite supercells with different point defects

Table 2. The ab initio lattice dynamics calculated fractionations between isotope Li species adopted in the forsterite structures via the interstitial and vacancy substitution mechanisms.

Table 3. Migration energies for Li vacancy and interstitial diffusions in forsterite. $\mathrm{Mg}$ vacancy migration energies are also given. *Indicates value from Ref. WALKER et al., 2009 
Figure Legends

Figure 1. (a) Lithium defects and migration pathways in forsterite lattice, viewed along [100], see text for details. (b) - (d). Li diffusion in forsterite via the mixed vacancy/interstitial mechanism looking through three directions (b) [100] (c) [010] and (d) [001]

Figure 2. Energy profiles for Lithium diffusion by hops A, B, C, and D (top); and the combined interstitial hops $E$ (from step 0 to step 10) and $F_{v}$ (from step 10 to step 22) (Bottom). The insert is the in-detailed plot of hop E. 
Table 1. Lattice energies for the perfect unit cells and the forsterite supercells with different point defects

\begin{tabular}{|c|c|c|}
\hline & $E(e V)$ & $\mathrm{E}_{\text {corr }}(\mathrm{eV})$ \\
\hline$V_{M g 1}^{\prime \prime}$ & $-90,906.37$ & $-90,904.48$ \\
\hline$V_{M g 2}^{\prime \prime}$ & $-90,905.34$ & $-90,903.45$ \\
\hline$L i_{i}^{*}$ & $-92,102.08$ & $-92,101.61$ \\
\hline$L i_{M g 1}^{\prime}$ & $-91,107.09$ & $-91,106.62$ \\
\hline$L i_{M g 2}^{\prime}$ & $-91,106.60$ & $-91,106.13$ \\
\hline$\left[L i_{i}^{*}-V_{M g 1}^{\prime \prime}-L i_{i}^{*}\right]_{S p l i t}$ & $-91,300.77$ & - \\
\hline$\left[L i_{i}^{*}-V_{M_{g} 2}^{\prime \prime}-L i_{i}^{*}\right]_{S p l i t}$ & $-91,300.20$ & - \\
\hline Forsterite $\left(\mathrm{Mg}_{2} \mathrm{SiO}_{4}\right)$ & $-15,317.14$ & \\
\hline
\end{tabular}

Notes: Defects are described using Kröger-Vink defect notation and include vacancies in both magnesium sites, a lithium interstitial and two lithium aliovalent substitutions, where Lithium ions occupying both free octahedral sites on both magnesium sites and two defects complex of split lithium interstitial across both magnesium sites. $\mathrm{E}_{\text {corr }}$ is the energy corrected for Columbic interactions between defects in neighboring unit cells. 
Table 2 The ab initio lattice dynamics calculated fractionations between isotope Li species adopted in the forsterite structures via the interstitial and vacancy substitution mechanisms.

\begin{tabular}{|l|l|l|l|l|}
\hline \multirow{2}{*}{\begin{tabular}{c} 
Temperature \\
\multirow{2}{*}{}
\end{tabular}} & \multicolumn{2}{|c|}{$\begin{array}{c}\text { Mass-dependent isotope } \\
\text { partition function ratio. }\end{array}$} & $\begin{array}{c}\text { Isotope fractionation } \\
\text { factor } \alpha_{\text {inter-vac }}\end{array}$ & $\Delta_{\text {inter-vac }}(\%)$ \\
\cline { 2 - 5 } & \multicolumn{1}{|c|}{$\beta_{\text {interstital }}$} & $\beta_{\text {vacancy }}$ & & \\
\hline 300 & 1.12510 & 1.03497 & 1.0871 & 87.1 \\
\hline 400 & 1.07262 & 1.02005 & 1.0515 & 8.8 \\
\hline 1000 & 1.01210 & 1.00328 & 1.0088 & 1.0 \\
\hline 3000 & 1.00135 & 1.00037 & 1.0010 & 8.8 \\
\hline$\Delta_{\text {inter-vac }}(\%)=\left(\alpha_{\text {inter-vac }}-1\right) \times 1000 \% 0$ & & \\
\hline
\end{tabular}


Table 3. Migration energies for Li vacancy and interstitial diffusions in forsterite. $\mathrm{Mg}$ vacancy migration energies are also given. *Indicates value from Ref. WALKER et al., 2009

\begin{tabular}{|l|c|c|c|}
\hline \multicolumn{2}{|l|}{} & \multicolumn{2}{|c|}{$\begin{array}{c}\text { Migration Activation energy } \\
\mathrm{kJmol}^{-1}\end{array}$} \\
\hline \multirow{4}{*}{ Vacancy diffusion } & $\mathrm{A}$ & 59.8 & $141.8^{*}$ \\
\cline { 2 - 4 } & $\mathrm{B}$ & 53.0 & $191.0^{*}$ \\
\cline { 2 - 4 } & $\mathrm{C}$ & 80.1 & $438.0^{*}$ \\
\cline { 2 - 5 } & $\mathrm{D}$ & 21.3 & 65.6 \\
\hline \multirow{3}{*}{$\begin{array}{l}\text { Interstitial diffusion } \\
\text { Vacancy assisted }\end{array}$} & $\mathrm{E}$ & 3.8 & $\left(69.4^{*}\right)$ \\
\cline { 2 - 5 } & $\mathrm{F}$ & 518.3 & \\
\hline interstitial diffusion & $\mathrm{F}$ & 55.9 & \\
\hline
\end{tabular}




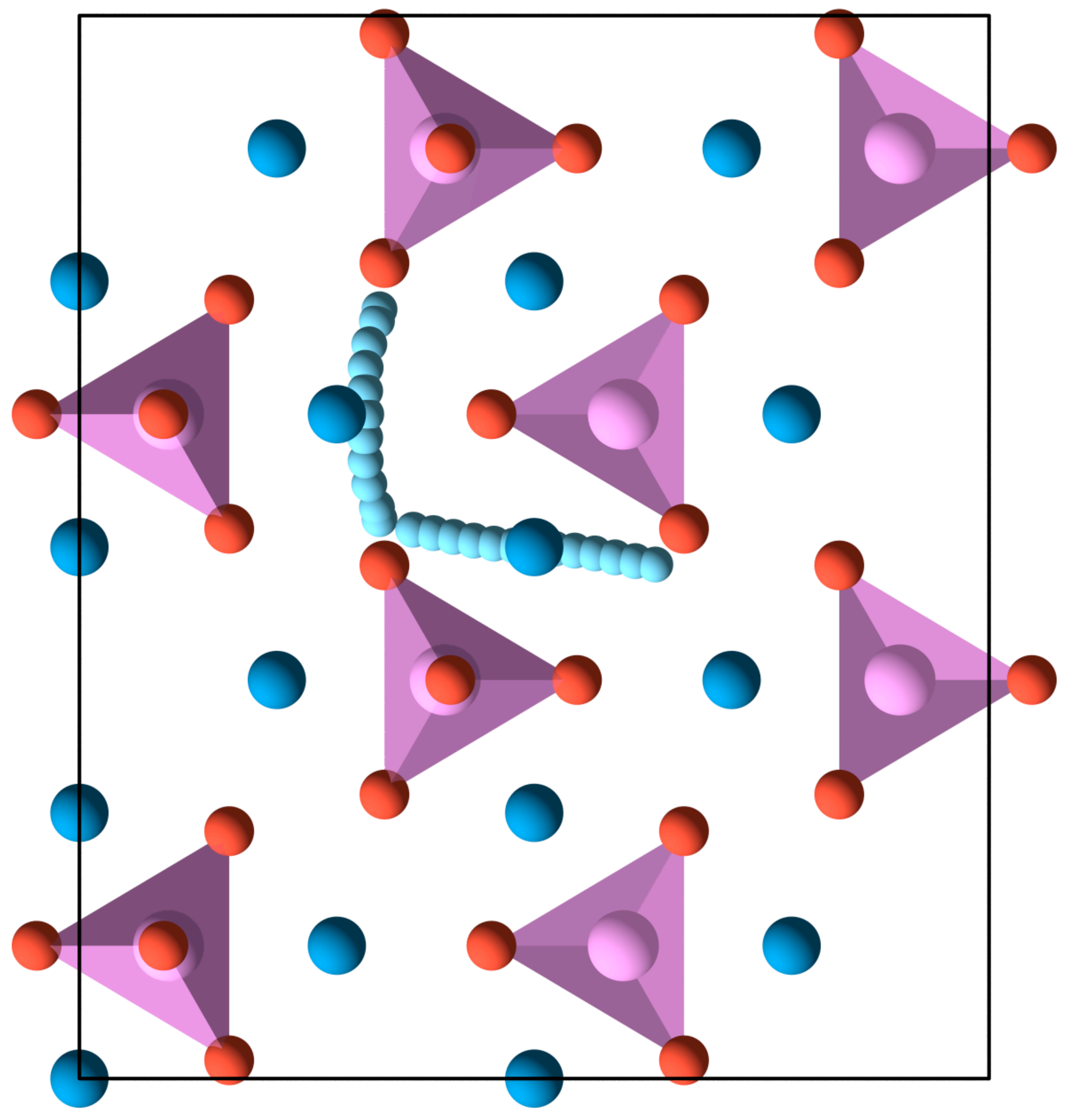




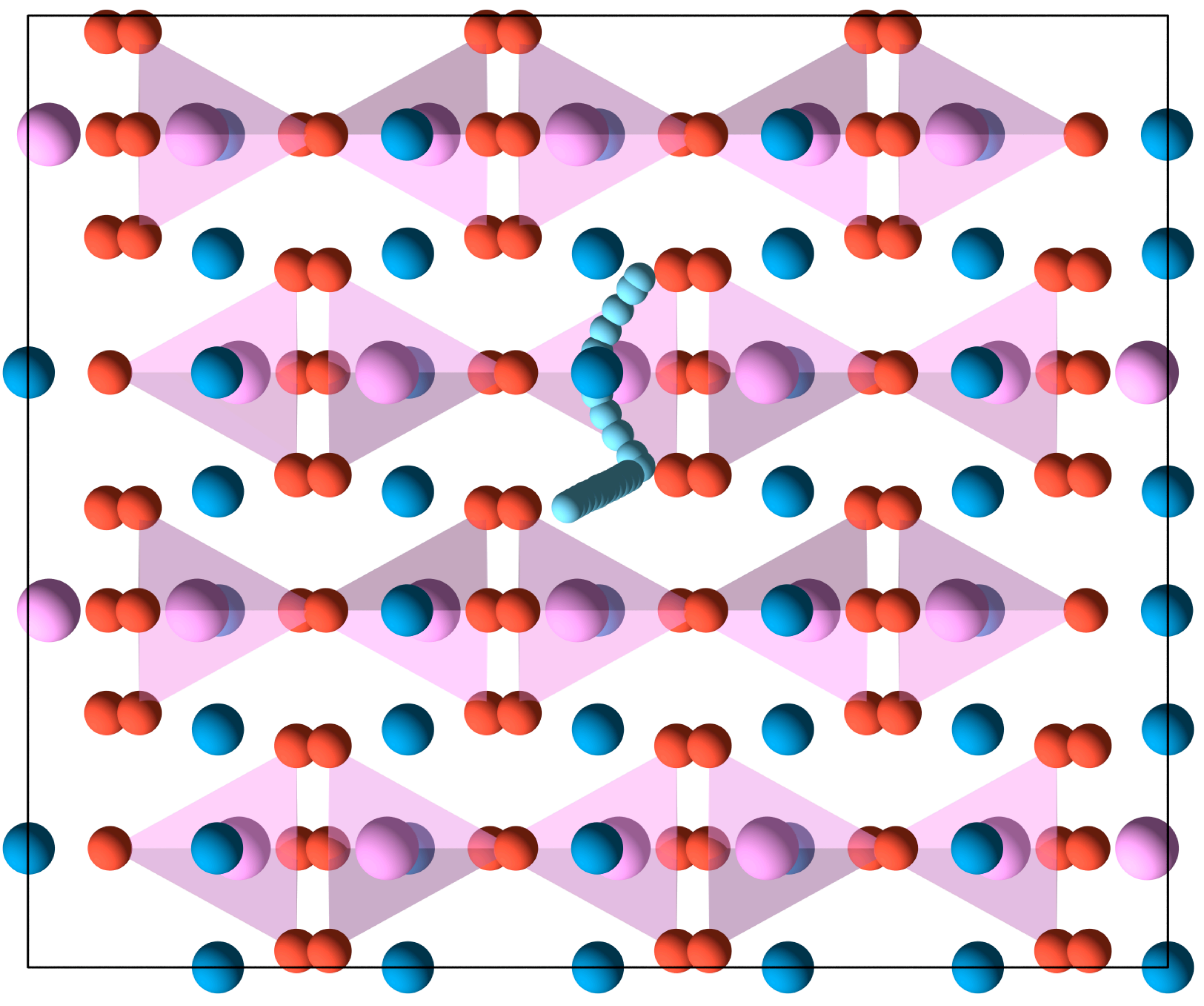




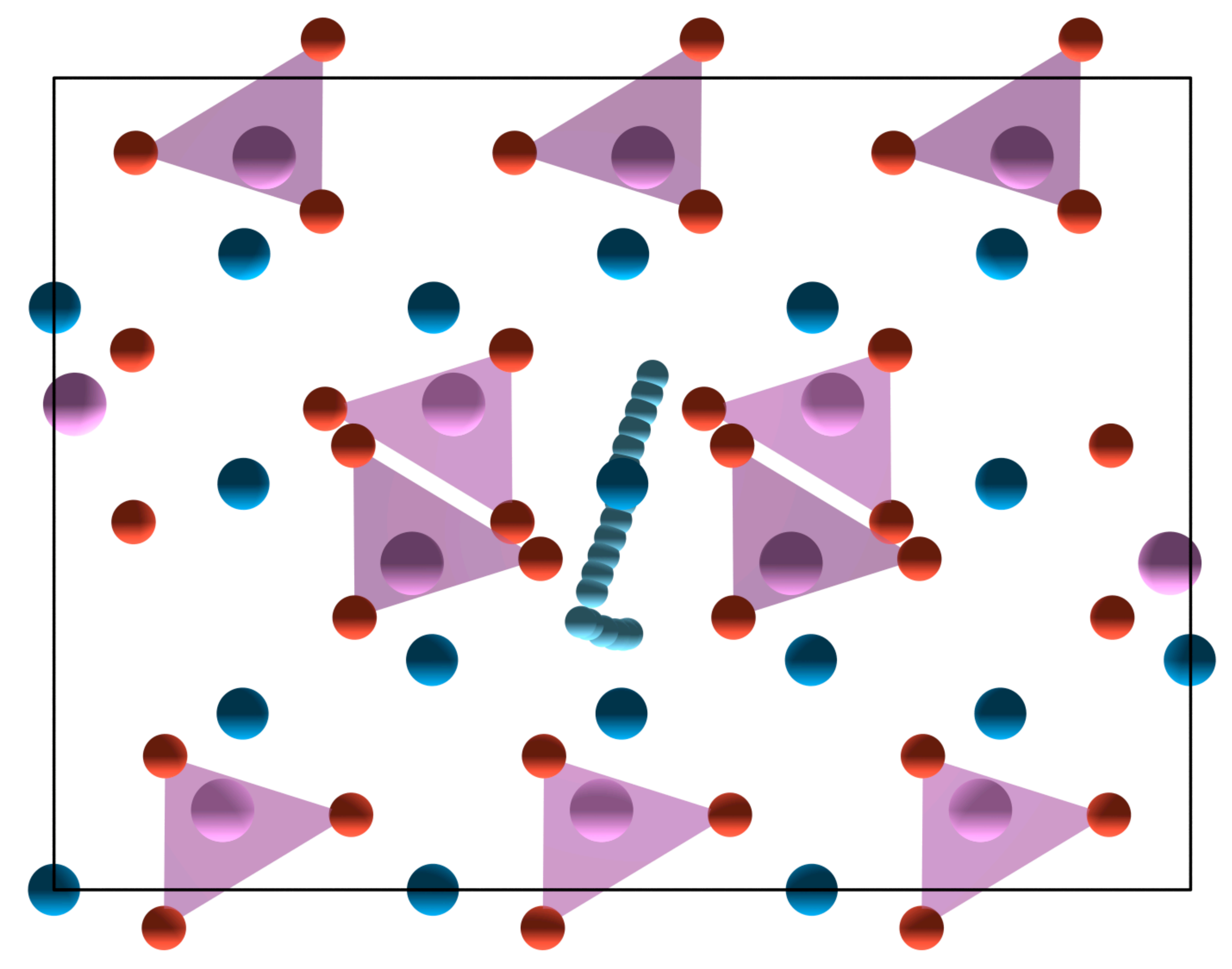



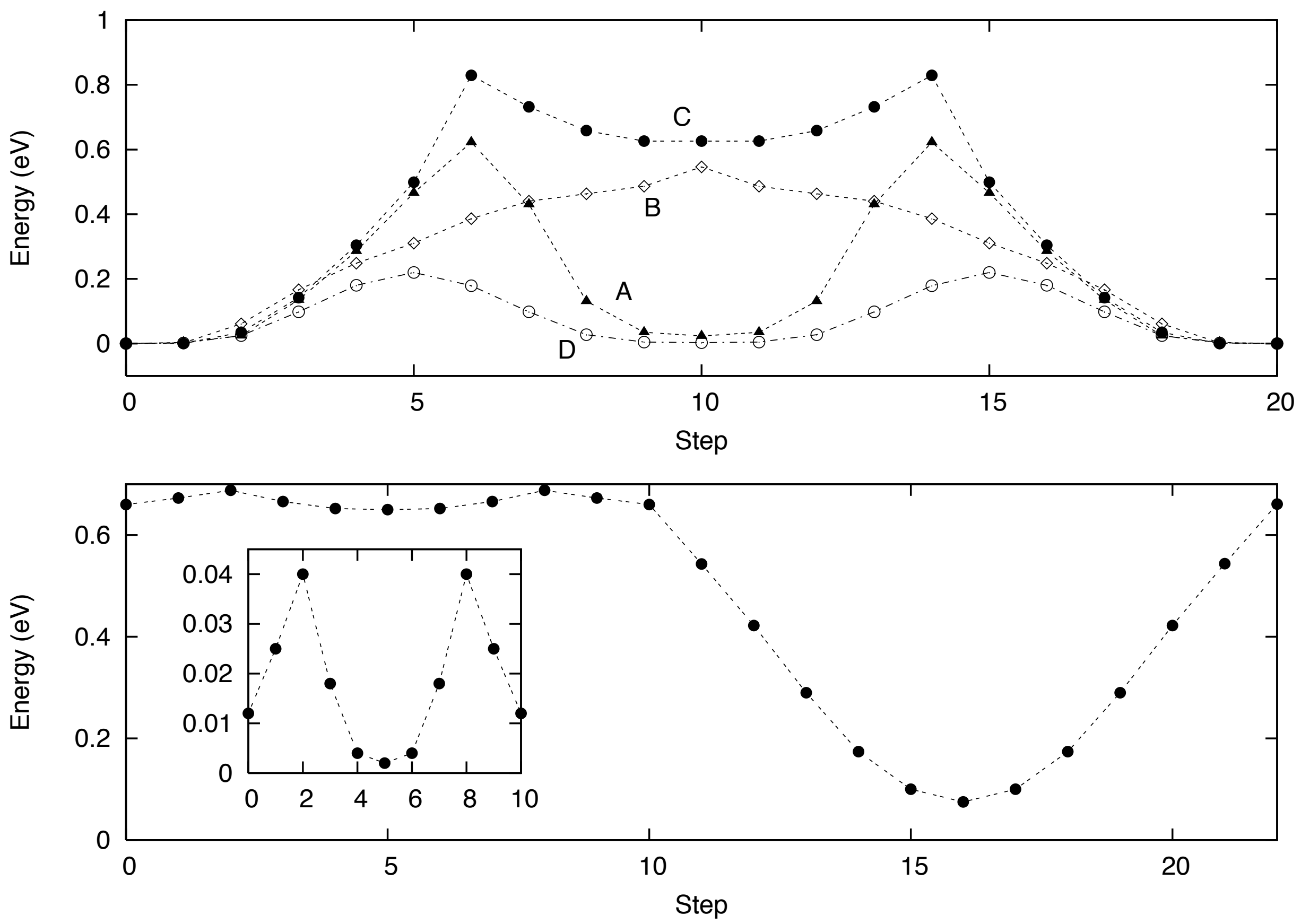